\title{
Average Output SINR of Equal-Gain Diversity in Correlated Nakagami- $m$ Fading With Cochannel Interference
}

\author{
Nikos C. Sagias, Member, IEEE, George K. Karagiannidis, Senior Member, IEEE, \\ Dimitris A. Zogas, Member, IEEE, George S. Tombras, Senior Member, IEEE, and Stavros A. Kotsopoulos
}

\begin{abstract}
In mobile radio systems, antenna diversity is used to combat fading and reduce the impact of cochannel interference. In this paper, we analyze the performance of $L$-branch equalgain combining receivers over correlated nonidentically distributed Nakagami- $m$ fading channels, in the presence of multiple identical cochannel interferers and additive white Gaussian noise. The performance criterion considered is the average output signalto-interference-plus-noise ratio, which is obtained in closed form for both independent and correlative fading. Due to the simple form of the derived expressions, they readily allow numerical evaluation for cases of practical interest.
\end{abstract}

Index Terms-Cochannel interference (CCI), correlative fading, equal-gain combining (EGC), Nakagami- $m$ fading, signal-tointerference-plus-noise ratio (SINR).

\section{INTRODUCTION}

C OCHANNEL INTERFERENCE (CCI) due to frequency reuse and multipath fading due to multipath propagation are the main causes for the performance degradation of cellular mobile radio systems. Diversity combining is a well-known method that can be used to alleviate the effects of these degradations. Some of the most well-known diversity techniques are maximal-ratio combining (MRC), equal-gain combining (EGC), and selection combining. Among them, EGC presents significant practical interest, because it provides performance comparable to MRC but with simpler implementation complexity. In EGC, the desired signals at the outputs of the $L$ antennas are cophased, equally weighted, and then summed to give the resultant desired signal.

Previous work concerning predetection EGC in the presence of CCI includes the following. Abu-Dayya and Bealieu have analytically derived the outage probability of EGC with CCI over independent Nakagami- $m$ fading channels [1]. Shah and Haimovich [2], assuming that the power of the interferers is equally distributed, have derived an expression for the ratio of the mean signal power to the mean interference power for the cases of a desired signal subject to Rayleigh or Rice fading and a CCI subject to Rice fading. In another work dealing with

Manuscript received October 1, 2003; revised March 1, 2004; accepted August 1,2004. The editor coordinating the review of this paper and approving it for publication is $\mathrm{H}$. Xu.

N. C. Sagias and G. S. Tombras are with the Department of Physics, University of Athens, 15784 Athens, Greece (e-mail: nsagias@space.noa.gr; gtombras@cc.uoa.gr).

G. K. Karagiannidis is with the Department of Electrical and Computer Engineering, Aristotle University of Thessaloniki, 54124 Thessaloniki, Greece (e-mail: geokarag@auth.gr).

D. A. Zogas and S. A. Kotsopoulos are with the Electrical and Computer Engineering Department, University of Patras, 26442 Patras, Greece (e-mail: zogas@space.noa.gr; kotsop@ee.upatras.gr).

Digital Object Identifier 10.1109/TWC.2005.850384
EGC receivers, Ma et al. [3] have studied the error performance of differential binary phase-shift keying in correlated Rician fading channels in the presence of CCI. More recently, Song et al. [4] have studied the outage performance of EGC over flat Rayleigh fading with multiple cochannel interferers in an interference-limited environment. However, to the best of the authors' knowledge, a useful expression for the average output signal-to-interference-plus-noise ratio (SINR) of EGC receivers in Nakagami- $m$ fading in both multiple $\mathrm{CCI}$ and additive white Gaussian noise (AWGN) has not been investigated yet for both independent and correlative fading. This is probably due to the lack of an analytical expression for the probability density function (pdf) of the sum of $L$ Nakagami- $m$ correlated fading envelopes and consequently of the pdf of the EGC output SINR.

In this paper, avoiding the difficulty of finding the latter pdf and using a simple and straightforward approach, analytical closed-form expressions for the average output SINR of $L$-branch predetection EGC receivers are derived. Our analysis includes both independent and correlative Nakagami- $m$ fading channels, in the presence of multiple equal average power Nakagami- $m$ cochannel interferers and AWGN. Numerical results show the effect of several parameters, such as the number and the average power of interferers, the correlation among the EGC branches, and the severity of the fading, on the combiner's performance.

The remainder of this paper is organized as follows. In Section II, the system and channel model are presented. In Section III, closed-form expressions for the EGC output SINR are derived. Several numerical results are included in Section IV, while Section V concludes the paper.

\section{System And Channel Model}

We consider a wireless communication system where the signal received from a desired user, over a total of $L$ diversity branches, is corrupted by $N$ cochannel interferers and AWGN. Assuming different interfering signals in each diversity branch, the complex baseband signal, at the input of the EGC receiver, can be conveniently expressed as

$$
\mathbf{z}=\mathbf{a} s+\sum_{i=1}^{N} \mathbf{r}_{i} d_{i}+\mathbf{w}
$$

where $\mathbf{z}=\left[\begin{array}{llll}z_{1} & z_{2} & \cdots & z_{L}\end{array}\right]^{\dagger}$ is the received signal matrix, with $[\cdot]^{\dagger}$ denoting the transpose matrix

$$
z_{\ell}=a_{\ell} s+\sum_{i=1}^{N} r_{i, \ell} d_{i}+w_{\ell}
$$


represents the received signal at the $\ell$ th antenna $(\ell=1$, $2, \ldots, L), r_{n, \ell}$ is the channel impulse response of the $n$th interferer $(n=1,2, \ldots, N)$, and $s$ and $d_{n}$ are the transmitted data symbols of the desired and the $n$th interfering signal, respectively. The vector $\mathbf{a}=\left[\begin{array}{llll}a_{1} & a_{2} & \cdots & a_{L}\end{array}\right]^{\dagger}$ is the channel matrix of the desired signal, $\mathbf{r}_{n}=\left[\begin{array}{llll}r_{n, 1} & r_{n, 2} & \cdots & r_{n, L}\end{array}\right]^{\dagger}$ is the channel matrix of the interfering signals, and $\mathbf{w}=\left[\begin{array}{llll}w_{1} & w_{2} & \cdots & w_{L}\end{array}\right]^{\dagger}$ is the AWGN matrix with a single-sided power spectral density $N_{0}$, identical for all branches. We assume that the interfering signals are uncorrelated with the desired signal and the interfering signal's phases are uniformly distributed in $[0,2 \pi)$. The instantaneous output SINR per symbol of the EGC receiver is

$$
\zeta=\frac{E_{\mathrm{s}}\left(\sum_{i=1}^{L}\left|a_{i}\right|\right)^{2}}{L N_{0}+E_{\mathrm{s}} \sum_{l=1}^{N}\left|\sum_{k=1}^{L} \widetilde{r}_{k, l}\right|^{2}}
$$

where $\widetilde{r}_{n, \ell}=r_{n, \ell} a_{\ell}^{*} /\left|a_{\ell}\right|$ and $a_{\ell}^{*}$ denote the complex conjugate of $a_{\ell}$. Moreover, the phases are uncorrelated with each other and with the interfering signal's amplitudes. Hence, the EGC receiver adds the desired signals coherently and the interfering signals incoherently [1]. Using this approximation, $\zeta$ can be expressed as

$$
\zeta \simeq \frac{E_{\mathrm{s}}\left(\sum_{i=1}^{L}\left|a_{i}\right|\right)^{2}}{L N_{0}+E_{\mathrm{s}} \sum_{l=1}^{N} \sum_{k=1}^{L}\left|r_{k, l}\right|^{2}}=\frac{\left(\sum_{i=1}^{L} \sqrt{\gamma_{i}}\right)^{2}}{L+\sum_{l=1}^{N} \sum_{k=1}^{L} R_{k, l}}
$$

where

$$
R_{n, \ell}=\left|r_{n, \ell}\right|^{2} \frac{E_{\mathrm{s}}}{N_{0}}
$$

is the instantaneous interference-to-noise power ratio (INR) per symbol of the $n$th interferer received in the $\ell$ th antenna and

$$
\gamma_{\ell}=\left|a_{\ell}\right|^{2} \frac{E_{\mathrm{s}}}{N_{0}}
$$

is the instantaneous SNR per symbol of the $\ell$ th branch with $E_{\mathrm{s}}=E\left\langle\left|d_{n}\right|^{2}\right\rangle=E\left\langle|s|^{2}\right\rangle$ being the transmitted symbol energy, whereby $E\langle\cdot\rangle$ statistical averaging is denoted. Assuming that both $\left|a_{\ell}\right|$ and $\left|r_{n, \ell}\right|$ are modeled as Nakagami-m random variables (RVs), the instantaneous SNR per symbol in the $\ell$ th branch $\gamma_{\ell}$ is Gamma distributed with a pdf given by [5, eq. (2.21)]

$$
p_{\gamma_{\ell}}(\gamma)=\left(\frac{m_{\ell}}{\bar{\gamma}_{\ell}}\right)^{m_{\ell}} \frac{\gamma^{m_{\ell}-1}}{\Gamma\left(m_{\ell}\right)} \mathrm{e}^{\frac{-\gamma m_{\ell}}{\bar{\gamma}_{\ell}}}
$$

where $\Gamma(\cdot)$ is the Gamma function [6, eq. (8.310/1)], $\bar{\gamma}_{\ell}$ is the average SNR per symbol of the desired component of the received signal, and $m_{\ell}$ is the corresponding Nakagami- $m$ fading parameter. The $\nu$ th moment of $\gamma_{\ell}$ is [5, eq. (2.23)]

$$
E\left\langle\gamma_{\ell}^{\nu}\right\rangle=\frac{\Gamma\left(m_{\ell}+\nu\right)}{\Gamma\left(m_{\ell}\right) m_{\ell}^{\nu}} \bar{\gamma}_{\ell}^{\nu}
$$

Having assumed that the interferers are mutually independent, the sum of Gamma identically distributed (i.d.) RVs with the same average INR per symbol $\bar{\gamma}_{I}$ and Nakagami- $m$ fading parameter $m_{\mathrm{I}}$, appearing in the denominator of (3), can be represented by another Gamma RV [7], i.e.,

$$
R=\sum_{k=1}^{N} \sum_{l=1}^{L} R_{k, l}
$$

with a pdf given by

$$
p_{R}(r)=\left(\frac{m_{\mathrm{I}}}{\bar{\gamma}_{\mathrm{I}}}\right)^{m_{\mathrm{I}} N L} \frac{r^{m_{\mathrm{I}} N L-1}}{\Gamma\left(m_{\mathrm{I}} N L\right)} \mathrm{e}^{-\frac{r m_{\mathrm{I}}}{\bar{\gamma}_{\mathrm{I}}}}
$$

which is equivalent to a single Nakagami- $m$ interferer with fading parameter $m_{\mathrm{I}} N L$ and average INR per symbol $\bar{\gamma}_{\mathrm{I}} N L$. Thus, (3) can be expressed as

$$
\zeta=\frac{1}{L+R}\left(\sum_{i=1}^{L} \sqrt{\gamma_{i}}\right)^{2} .
$$

\section{Average OUtPut SINR}

The average output SINR per symbol of the EGC can be obtained by averaging (10), i.e.,

$$
\bar{\zeta}=E\left\langle\frac{1}{L+R}\left(\sum_{i=1}^{L} \sqrt{\gamma_{i}}\right)^{2}\right\rangle .
$$

Since the desired signal and the interferers are mutually independent, (11) can be written as

$$
\bar{\zeta}=E\left\langle\frac{1}{L+R}\right\rangle E\left\langle\left(\sum_{i=1}^{L} \sqrt{\gamma_{i}}\right)^{2}\right\rangle .
$$

The two mean terms in (12) can be evaluated separately. The first mean term $E\langle 1 /(L+R)\rangle$ can be derived using (9) as

$$
E\left\langle\frac{1}{L+R}\right\rangle=\int_{0}^{\infty} \frac{1}{L+r} p_{R}(r) \mathrm{d} r
$$

which, using [6, eq. (3.383/10)] can be expressed as

$$
E\left\langle\frac{1}{L+R}\right\rangle=\frac{1}{L}\left(L \frac{m_{\mathrm{I}}}{\bar{\gamma}_{\mathrm{I}}}\right)^{m_{\mathrm{I}} N L} \mathrm{e}^{\frac{L m_{\mathrm{I}}}{\bar{\gamma}_{\mathrm{I}}}} \Gamma\left(1-m_{\mathrm{I}} N L, L \frac{m_{\mathrm{I}}}{\bar{\gamma}_{\mathrm{I}}}\right)
$$

where $\Gamma(\cdot, \cdot)$ is the upper incomplete Gamma function [6, eq. (8.350/2)]. The second mean term $E\left\langle\left(\sum_{i=1}^{L} \sqrt{\gamma_{i}}\right)^{2}\right\rangle$ in (12) can be derived using the multinomial identity [8, eq. (24.1.2)] by expanding the squared sum term $\left(\sum_{i=1}^{L} \sqrt{\gamma}_{i}\right)^{2}$, yielding

$$
E\left\langle\left(\sum_{i=1}^{L} \sqrt{\gamma}_{i}\right)^{2}\right\rangle=2 \sum_{\substack{k_{1}, k_{2}, \ldots, k_{L}=0 \\ k_{1}+k_{2}+\cdots+k_{L}=2}}^{2} E\left\langle\prod_{j=1}^{L} \frac{\gamma_{j}^{\frac{k_{j}}{2}}}{k_{j} !}\right\rangle
$$


and thus, the average output SINR per symbol of the EGC diversity receiver can be written as

$$
\begin{aligned}
\bar{\zeta}=\frac{2}{L}\left(L \frac{m_{\mathrm{I}}}{\bar{\gamma}_{\mathrm{I}}}\right)^{m_{\mathrm{I}} N L} \mathrm{e}^{\frac{L m_{\mathrm{I}}}{\bar{\gamma}_{\mathrm{I}}}} \Gamma\left(1-m_{\mathrm{I}} N L, L \frac{m_{\mathrm{I}}}{\bar{\gamma}_{\mathrm{I}}}\right) \\
\\
\times \sum_{\substack{k_{1}, k_{2}, \ldots, k_{L}=0 \\
k_{1}+k_{2}+\cdots+k_{L}=2}}^{2} E\left\langle\prod_{j=1}^{L} \frac{\gamma_{j}^{\frac{k_{j}}{2}}}{k_{j} !}\right\rangle .
\end{aligned}
$$

\section{A. Independent Fading}

For independent, but not necessarily i.d. input paths, the term $E\left\langle\prod_{j=1}^{L} \gamma_{j}^{k_{j} / 2}\right\rangle$ in (16) can be expressed using (7) as

$$
E\left\langle\prod_{j=1}^{L} \gamma_{j}^{\frac{k_{j}}{2}}\right\rangle=\prod_{j=1}^{L} E\left\langle\gamma_{j}^{\frac{k_{j}}{2}}\right\rangle=\prod_{j=1}^{L} \frac{\Gamma\left(m_{j}+\frac{k_{j}}{2}\right)}{\Gamma\left(m_{j}\right) m_{j}^{\frac{k_{j}}{2}}} \bar{\gamma}_{j}^{\frac{k_{j}}{2}}
$$

By replacing (17) in (16), the average output SINR per symbol of the EGC, with independent input paths, can be written as

$$
\begin{aligned}
\bar{\zeta}=\frac{2}{L}\left(L \frac{m_{\mathrm{I}}}{\bar{\gamma}_{\mathrm{I}}}\right)^{m_{\mathrm{I}} N L} \mathrm{e}^{\frac{L m_{\mathrm{I}}}{\bar{\gamma}_{\mathrm{I}}}} \Gamma\left(1-m_{\mathrm{I}} N L, L \frac{m_{\mathrm{I}}}{\bar{\gamma}_{\mathrm{I}}}\right) \\
\times \sum_{\substack{k_{1}, k_{2}, \ldots, k_{L}=0 \\
k_{1}+k_{2}+\cdots+k_{L}=2}}^{2} \prod_{j=1}^{L} \frac{\Gamma\left(m_{j}+\frac{k_{j}}{2}\right)}{k_{j} ! \Gamma\left(m_{j}\right) m_{j}^{\frac{k_{j}}{2}}} \bar{\gamma}_{j}^{\frac{k_{j}}{2}}
\end{aligned}
$$

which after straightforward algebraic manipulation can be expressed in a simple and closed form as

$$
\begin{aligned}
\bar{\zeta}= & \frac{1}{L}\left(L \frac{m_{\mathrm{I}}}{\bar{\gamma}_{\mathrm{I}}}\right)^{m_{\mathrm{I}} N L} \mathrm{e}^{\frac{L m_{\mathrm{I}}}{\bar{\gamma}_{\mathrm{I}}}} \Gamma\left(1-m_{\mathrm{I}} N L, L \frac{m_{\mathrm{I}}}{\bar{\gamma}_{\mathrm{I}}}\right) \\
& \times\left[\sum_{i=1}^{L} \bar{\gamma}_{i}+2 \sum_{i=2}^{L} \sum_{j=1}^{i-1} \frac{\Gamma\left(m_{i}+\frac{1}{2}\right) \Gamma\left(m_{j}+\frac{1}{2}\right)}{\Gamma\left(m_{i}\right) \Gamma\left(m_{j}\right)} \sqrt{\frac{\bar{\gamma}_{i} \bar{\gamma}_{j}}{m_{i} m_{j}}}\right] .
\end{aligned}
$$

For i.d. input paths $\left(\bar{\gamma}_{\ell}=\bar{\gamma}_{0}\right.$ and $\left.m_{\ell}=m\right)$, (19) reduces to

$$
\begin{aligned}
\bar{\zeta}=\bar{\gamma}_{0}\left(L \frac{m_{\mathrm{I}}}{\bar{\gamma}_{\mathrm{I}}}\right)^{m_{\mathrm{I}} N L} \mathrm{e}^{\frac{L m_{\mathrm{I}}}{\overline{\bar{\gamma}}_{\mathrm{I}}}} \Gamma\left(1-m_{\mathrm{I}} N L, L \frac{m_{\mathrm{I}}}{\bar{\gamma}_{\mathrm{I}}}\right) \\
\times\left[1+(L-1) \frac{\Gamma^{2}\left(m+\frac{1}{2}\right)}{m \Gamma^{2}(m)}\right] .
\end{aligned}
$$

\section{B. Correlative Fading}

For correlative input paths, (17) does not hold and hence the term $E\left\langle\prod_{j=1}^{L} \gamma_{j}^{k_{j} / 2}\right\rangle$ has to be evaluated taking into account the correlation among $a_{1}, a_{2}, \ldots, a_{L}$. However, it can be easily recognized that the only unknown terms that appear in (16) are of the form of $E\left\langle\sqrt{\gamma_{i} \gamma_{j}}\right\rangle$. These terms can be obtained, using [9, eq. (137)]

$$
\begin{aligned}
& E\left\langle\gamma_{i}^{n_{i}} \gamma_{j}^{n_{j}}\right\rangle=\frac{\bar{\gamma}_{i}^{n_{i}} \bar{\gamma}_{j}^{n_{j}} \Gamma\left(m+n_{i}\right) \Gamma\left(m+n_{j}\right)}{m^{n_{i}+n_{j}} \Gamma^{2}(m)} \\
& \times{ }_{2} F_{1}\left(-n_{i},-n_{j} ; m ; \rho_{i, j}\right)
\end{aligned}
$$

with $m$ being the fading parameter of all paths, $\rho_{i, j}$ being the correlation coefficient among the $i$ th and $j$ th input paths ( $\rho_{i, j}=$ $\left.\rho_{j, i}, \rho_{i, i}=1, i, j=1,2, \cdots, L\right)$, and ${ }_{2} F_{1}(\cdot, \cdot ; \cdot ; \cdot)$ denoting the Gauss hypergeometric function of [6, eq. (9.100)]. Substituting (21) in (16), the average output SINR per symbol of the EGC diversity receiver, with arbitrary correlation between the input branches, can be obtained in a simple closed-form expression as

$$
\begin{aligned}
\bar{\zeta}=\frac{1}{L}\left(L \frac{m_{\mathrm{I}}}{\bar{\gamma}_{\mathrm{I}}}\right)^{m_{\mathrm{I}} N L} \mathrm{e}^{\frac{L m_{\mathrm{I}}}{\bar{\gamma}_{\mathrm{I}}}} \Gamma\left(1-m_{\mathrm{I}} N L, L \frac{m_{\mathrm{I}}}{\bar{\gamma}_{\mathrm{I}}}\right) \\
\times\left\{\sum_{i=1}^{L} \bar{\gamma}_{i}+\frac{2 \Gamma^{2}\left(m+\frac{1}{2}\right)}{m \Gamma^{2}(m)}\right. \\
\left.\quad \times \sum_{i=2}^{L} \sum_{j=1}^{i-1}\left[{ }_{2} F_{1}\left(-\frac{1}{2},-\frac{1}{2} ; m ; \rho_{i, j}\right) \sqrt{\bar{\gamma}_{i} \bar{\gamma}_{j}}\right]\right\} .
\end{aligned}
$$

Note that for uncorrelated input paths, $\rho_{i, j}=0(i \neq j)$, (22) reduces to (19) for $m_{\ell}=m$, since for this special value of $\rho_{i, j}$, ${ }_{2} F_{1}(-1 / 2,-1 / 2 ; m ; 0)=1$. Furthermore, for i.d. input paths, (22) further simplifies to

$$
\begin{aligned}
\bar{\zeta}= & \frac{\bar{\gamma}_{0}}{L}\left(L \frac{m_{\mathrm{I}}}{\bar{\gamma}_{\mathrm{I}}}\right)^{m_{\mathrm{I}} N L} \mathrm{e}^{\frac{L m_{\mathrm{I}}}{\bar{\gamma}_{\mathrm{I}}}} \Gamma\left(1-m_{\mathrm{I}} N L, L \frac{m_{\mathrm{I}}}{\bar{\gamma}_{\mathrm{I}}}\right) \\
& \times\left[L+\frac{2 \Gamma^{2}\left(m+\frac{1}{2}\right)}{m \Gamma^{2}(m)} \sum_{i=2}^{L} \sum_{j=1}^{i-1}{ }_{2} F_{1}\left(-\frac{1}{2},-\frac{1}{2} ; m ; \rho_{i, j}\right)\right] .
\end{aligned}
$$

To the best of the authors' knowledge, (19) and (22) are novel.

\section{Numerical RESUlts}

In this section, some numerical results for the average output SINR performance of the EGC receiver operating over Nakagami- $m$ fading in the presence of both CCI and AWGN are presented. Equations (19) and (22) have been numerically evaluated and the results are illustrated in Figs. 1-3. It is assumed that the receiver operates with an exponentially decaying power delay profile

$$
\bar{\gamma}_{\ell}=\bar{\gamma}_{1} \mathrm{e}^{-\delta(\ell-1)}
$$

with $\delta$ being the power decaying factor. In Fig. 1, the normalized, to the average SNR of the first input branch, average output SINR $\bar{\zeta} / \bar{\gamma}_{1}$ of EGC is plotted as a function of $L$ for $\bar{\gamma}_{\mathrm{I}}=0 \mathrm{~dB}, N=2, m_{\mathrm{I}}=0.75$, constant correlation $\rho_{i, j}=0.5$ among the input branches, and for several values of $m$ and $\delta$. It can be easily recognized that the combined loss of the receiver 


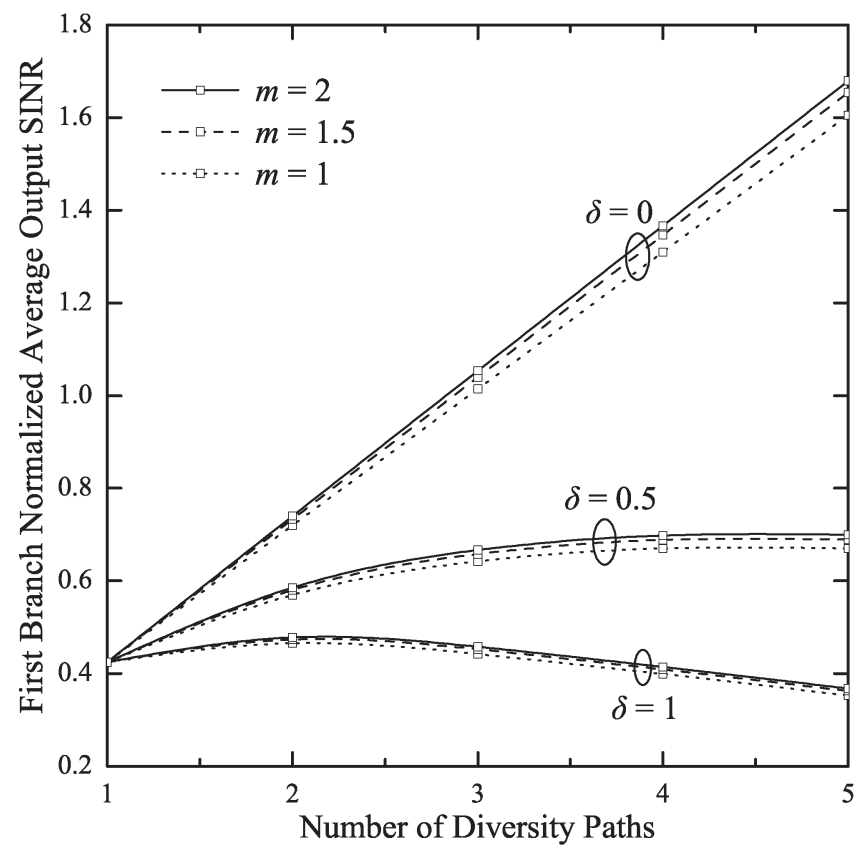

Fig. 1. First branch normalized average output $\operatorname{SINR} \bar{\zeta} / \bar{\gamma}_{1}$ of the EGC versus $L$ for exponentially decaying PDP and constant correlation among the branches $\left(\bar{\gamma}_{\mathrm{I}}=0 \mathrm{~dB}, N=2, m_{\mathrm{I}}=0.75\right.$, and $\left.\rho=0.5\right)$.

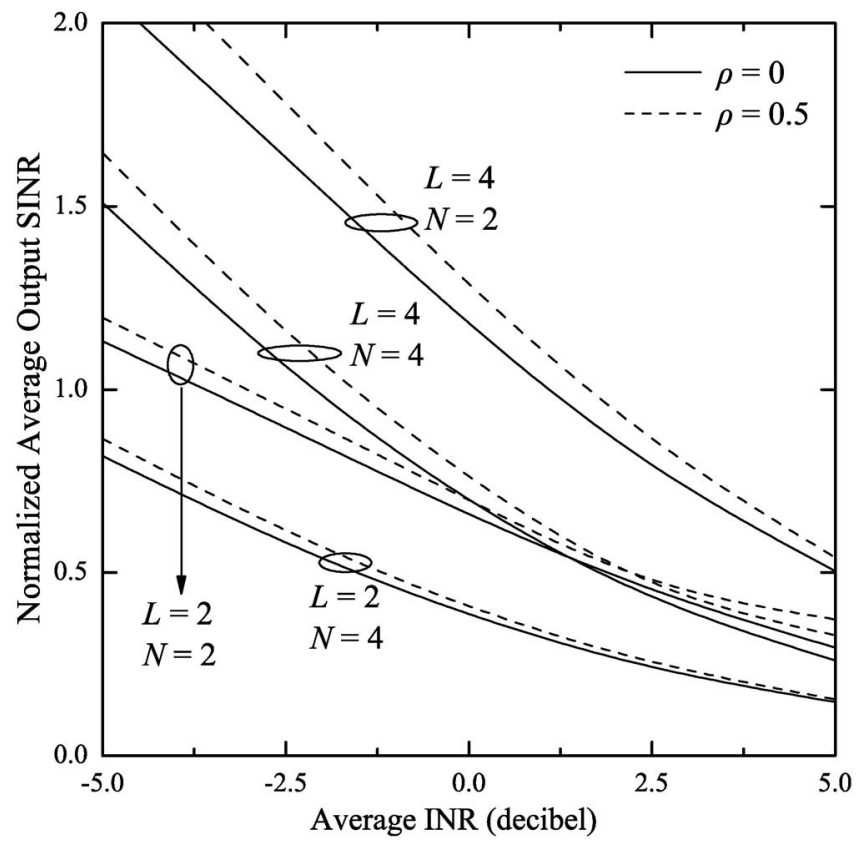

Fig. 2. Normalized average output SINR $\bar{\zeta} / \bar{\gamma}_{0}$ of the EGC versus $\bar{\gamma}_{\mathrm{I}}$ for exponential correlation among the branches $\left(m=m_{\mathrm{I}}=1\right.$ and $\rho=0$ and 0.5$)$.

gets more accentuated as $\delta$ increases. As expected, while the fading severity of the desired signal decreases, higher values for the normalized average output SINR are obtained.

In Fig. 2, the normalized, to the average input SNR, average output SINR of EGC is plotted for i.d. input branches as a function of $\bar{\gamma}_{\mathrm{I}}$ for independent and exponentially correlated input paths $\rho_{i, j}=\rho^{|i-j|}$ with $\rho=0.5, m=m_{\mathrm{I}}=1$, and for several values of $L$ and $N$. As shown, the EGC efficiently combats the interference as the number of diversity branches increases,

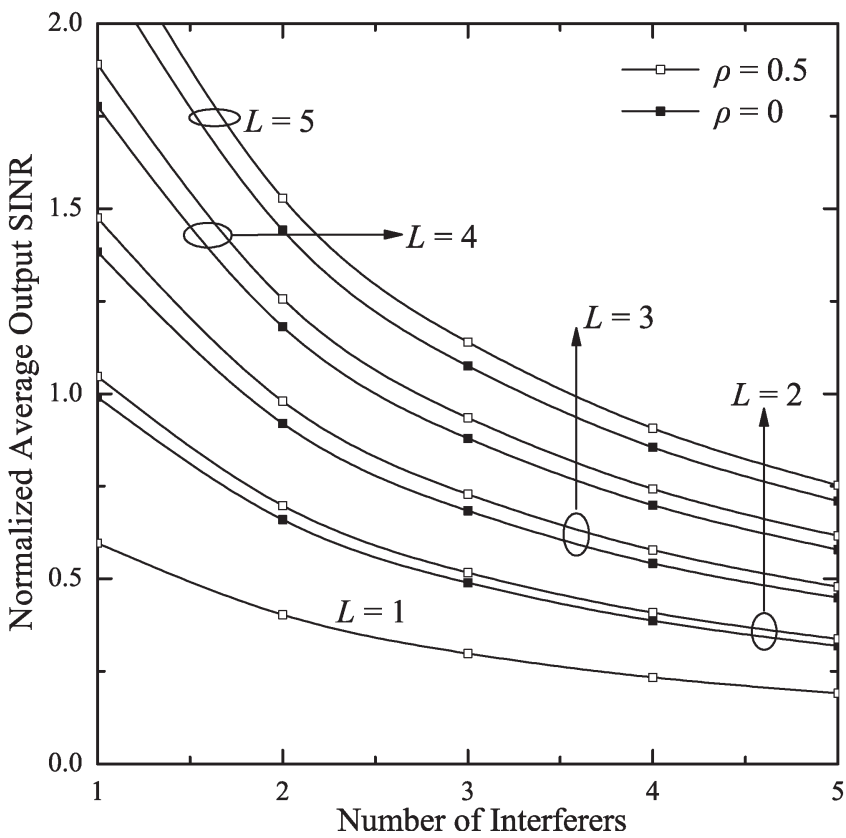

Fig. 3. Normalized average output SINR $\bar{\zeta} / \bar{\gamma}_{0}$ of the EGC versus $N$ for exponential correlation among the branches $\left(\bar{\gamma}_{\mathrm{I}}=0 \mathrm{~dB}, m=m_{\mathrm{I}}=1\right.$, and $\rho=0$ and 0.5 ).

while $\bar{\zeta} / \bar{\gamma}_{0}$ decreases with an increase of $\bar{\gamma}_{\mathrm{I}}$. Furthermore, $\bar{\zeta} / \bar{\gamma}_{0}$ increases as the correlation between the branches increases.

In Fig. 3, the normalized average output SINR $\bar{\zeta} / \bar{\gamma}_{0}$ of EGC is plotted for i.d. input branches as a function of $N$ for independent and exponentially correlated statistics, $\rho=0.5$, $\bar{\gamma}_{\mathrm{I}}=0 \mathrm{~dB}, m=m_{\mathrm{I}}=1$, and for several values of $L$. We observe that for an increase of $N$, a performance floor appears, due to the low fading severity of the composed interfering signal, so the diversity improvement diminishes. Moreover, $\bar{\zeta} / \bar{\gamma}_{0}$ increases as $\rho$ increases, as also observed in Fig. 2.

It must be noted here that (3) is a very good approximation of (2) and has also been used by other authors in the past [1], since the analytical derivation of the average EGC output SINR is very difficult, if not impossible. For comparison purposes, we have performed some Monte Carlo computer simulations for the exact average output SINR using (2). By comparing the simulations and evaluating results obtained using (2) and (3), respectively, it was realized that the approximation that has been considered is quite accurate. More specifically, computer simulation results were constantly at about 5\% lower than the corresponding analytical results. Therefore, they are not included in Figs. 1-3 for simplicity and readability purposes. Moreover, the computer simulation results can only be derived for integer values of the fading severity parameters, which would limit the generality of the proposed formulation.

\section{CONCLUSION}

We presented analytical closed-form expressions for the average output SINR for cellular mobile radio systems employing EGC diversity. The receiver operates over independent or correlated but not necessarily i.d. Nakagami- $m$ fading channels in the presence of multiple Nakagami- $m$ cochannel interferers 
and AWGN. In our proposed formulation, it was assumed that the desired signals are added coherently and the interfering signals are added incoherently across the antenna array elements. Numerical results clearly show the negative effect of several parameters, such as the average INR and the number of the interferers, on the average output SINR performance of the EGC, while computer simulation results verified the accuracy of the derived analytical results.

\section{ACKNOWLEDGMENT}

The authors would like to thank the anonymous reviewers for their careful reading and for many interesting remarks.

\section{REFERENCES}

[1] A. Abu-Dayya and N. C. Beaulieu, "Outage probabilities of diversity cellular systems with co-channel interference in Nakagami fading," IEEE Trans. Veh. Technol., vol. 41, no. 4, pp. 343-355, Nov. 1992.
[2] A. Shah and A. M. Haimovich, "Performance analysis of maximal ratio combining and comparison with optimum combining for mobile radio communications with co-channel interference," IEEE Trans. Commun., vol. 49, no. 4, pp. 1454-1463, Jul. 2000.

[3] Y. Ma, J. Lim, and S. Pasupathy, "Error probability for coherent and differential PSK over arbitrary Rician fading channels with multiple cochannel interferers," IEEE Trans. Commun., vol. 50, no. 3, pp. 429-441, Mar. 2002.

[4] Y. Song, S. D. Blostein, and J. Cheng, "Exact outage probability for equal gain combining with cochannel interference in Rayleigh fading," IEEE Trans. Wireless Commun., vol. 2, no. 5, pp. 865-870, Sep. 2003.

[5] M. K. Simon and M.-S. Alouini, Digital Communication Over Fading Channels, 1st ed. New York: Wiley, 2001.

[6] I. S. Gradshteyn and I. M. Ryzhik, Table of Integrals, Series, and Products, 6th ed. New York: Academic, 2000.

[7] A. Abu-Dayya and N. C. Beaulieu, "Outage probabilities of cellular mobile radio systems with multiple Nakagami interferers," IEEE Trans. Veh. Technol., vol. 40, no. 4, pp. 757-768, Nov. 1991.

[8] M. Abramovitz and I. A. Stegun, Handbook of Mathematical Functions With Formulas, Graphs, and Mathematical Tables, 9th ed. New York: Dover, 1972.

[9] M. Nakagami, The m-Distribution-A General Formula if Intensity Distribution of Rapid Fading, W. G. Hoffman, Ed. Oxford, U.K.: Pergamon, 1960. 